\title{
MECHANICAL ANALYSIS ON THE WORKING BEHAVIOUR OF MID-WATER TRAWL
}

\author{
Otohiko SuzukI* \\ (Received Aug. 9, 1961)
}

In order to obtain successful mid-water trawling, it will be a matter of concern to adjust the working depth of the net to any depth where fish have been located, and to keep the net mouth as large as possible.

The present study deals with the configuration of the mouth of the net, which is a simple model of elliptic cone type, from the mechanical point of view. Generally, it is too complicated to discuss analytically the behaviour of fishing net in action. In our laboratory, an approximate method ${ }^{1,2,3)}$ for analysing such a problem has been developed. After the same principle, in this paper, the form of each part of the net is approximated to a straight line or to a curve, and the curve is deemed to be composed of one or two circular arcs of different radii. Thus the problem can be reduced to solve the simple equations of mechanical equilibrium. Solving these equations simultaneously, we can get the mechanical action and the working behaviour of the net.

It seems to be a tedious procedure to solve these problems by such a method. This method, however, has the advantage of calculating the forces and the tensions acting on the net, while these measurements are very difficult in the ordinary model test.

\section{Theoretical Considerations}

The mid-water trawl is commonly constructed of four pieces of tapered, or triangular webbing; and is, approximately, considered to form an elliptical cone under towing condition. A geometrical configuration of the net mouth is determined by two mechanical actions, i. e., a lateral spreading force, which is due to the horizontal component of towing force, and a vertical supporting force due to the float and sinker.

For the sake of simplicity, suppose the case where the buoyancy of float is neutralized by the weight of sinker, then the geometrical configuration and the mechanical action of the net may be symmetrical about the central axis of the elliptical cone. Thus, it will suffice to consider merely about the quarter part of the net, say the top, port side.

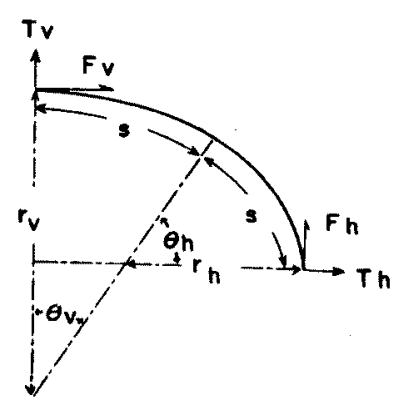

Fig. 1. Schematic represen. tation of the front view of top, port quarter of the net.

Briefly, let us suppose that the elliptical shape assumed

* Dept. of Fish., Kyoto Univ., Maizuru, Japan. (鈴木乙彦, 京大·水産) 
by the net mouth can be approximated to a combination of two circular arcs of different radii, $r_{v}, r_{n}$, and of equal length, $s$. This length, $s$, which is one-eighth of the circumference, $S$, of the net mouth may be written as

$$
s \equiv \frac{S}{8}=r_{v} \theta_{v}=r_{k} \theta_{\hbar},
$$

and

$$
\theta_{v}+\theta_{k}=\frac{\pi}{2}
$$

where, $\theta_{v}$ and $\theta_{h}$ are the central angles of arcs (Fig. 1). From the equilibrium between the spreading forces, $T_{v}$ and $T_{h}$, and the tensions, $F_{v}$ and $F_{k}$, acting on the circumference of the mouth, we get mechanically

$$
\begin{aligned}
& F_{v}=T_{v} r_{v}, \\
& F_{k}=T_{k} r_{n} .
\end{aligned}
$$

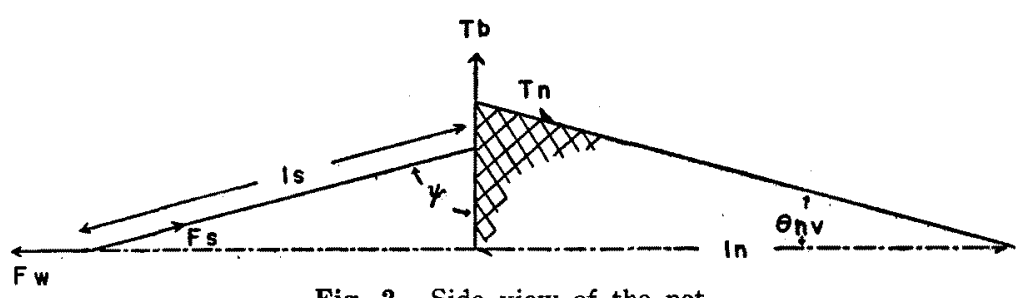

Fig. 2. Side view of the net.

It is assumed, here, that the buoyancy of float and the weight of sinker are constant irrespective of the towing speed. Let $T_{b}$ be the buoyancy or weight per unit length of the framing rope which forms the mouth of net, $T_{n}$ be the vertical com. ponent of resistance per unit length of the rope acting along the netting, and $\theta_{n v}$ be the angle between the top or lower piece of the net and the towing direction (Fig. 2). The vertical supporting force $T_{v}$ is expressed as

$$
T_{v}=T_{b}-T_{n} \sin \theta_{n v}
$$

In the above expression, $T_{n}$ is estimated by assuming that the shape of the net is a circular cone, and that the TAUTI's expression ${ }^{4}$ for resistance of netting can be tenable, i. e.,

$$
S T_{n}=k \frac{D}{L} \tan \varphi \cos \theta A V^{2}
$$

where, $D$ and $L$ are the diameter of the netting twine and the bar-length of the mesh respectively, $2 \varphi$ the angle between the adjacent bars of the mesh, $2 \theta$ the angle of the apex of the cone, $A$ the total area of webbing, $V$ the towing speed, and $k$ the coefficient of resistance of the netting twine.

Next, let $F_{w}$ be the tension of the warp, $\phi$ be the angle of the warp to the tow- 
ing direction, and $\theta_{n n}$ be the angle between the side piece of the net and the towing direction (Fig. 3). The horizontal spreading force $T_{k}$ is given by

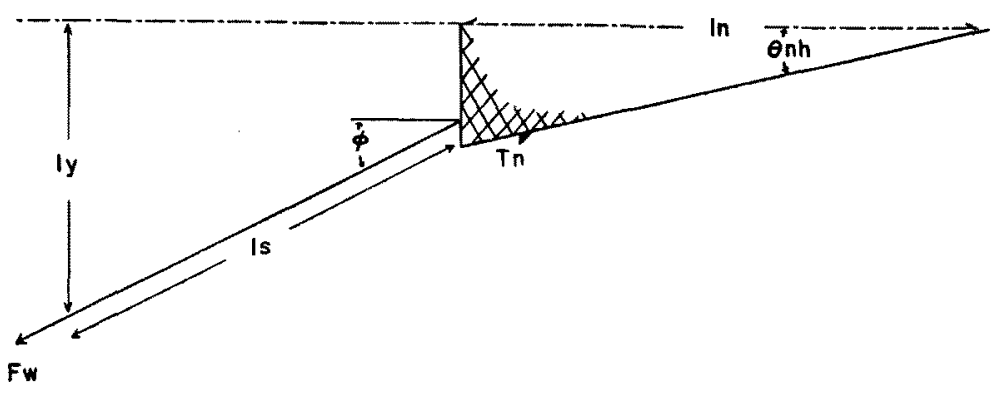

Fig. 3. Top view of the net.

$$
T_{k}=\frac{F_{w}}{2 s} \sin \phi-T_{n} \sin \theta_{n h} .
$$

From the equilibrium system of the towing force and the resistance of the net, we have

$$
F_{w} \cos \phi=2 s T_{n}\left(\cos \theta_{n k}+\cos \theta_{n v}\right) .
$$

According to geometrical relation, one half length of the vertical and the horizontal spreads of the mouth are respectively written as follows

$$
\begin{aligned}
& r_{v}-\left(r_{v}-r_{k}\right) \cos \theta_{v}=l_{n} \tan \theta_{n v}, \\
& r_{k}+\left(r_{b}-r_{k}\right) \sin \theta_{v}=l_{n} \tan \theta_{n h},
\end{aligned}
$$

where $l_{n}$ is the length of the net measured along the central axis.

Since the tension, $F_{w}$, in the warp is resolved into two tensions, $F_{s}$, on the two sweep lines, we have

$$
F_{w}=2 F_{s} \sin \phi,
$$

where $\phi$ is the angle between the sweep line and the vertical direction. Denoting the length of the sweep line by $l_{s}$, we have geometrically

$$
l_{8} \cos \psi=r_{h} \sin \theta_{h} \text {. }
$$

As regards the ropes which forms the net mouth, the tension, $F_{v}$, acting on the top or lower part of the framing rope is obtained by adding the component of tension, $F_{s}$, acting on the sweep line to the tension, $F_{h}$, of the side framing rope (Fig. 4). Thus the relation between $F_{h}$ and $F_{v}$ is given by

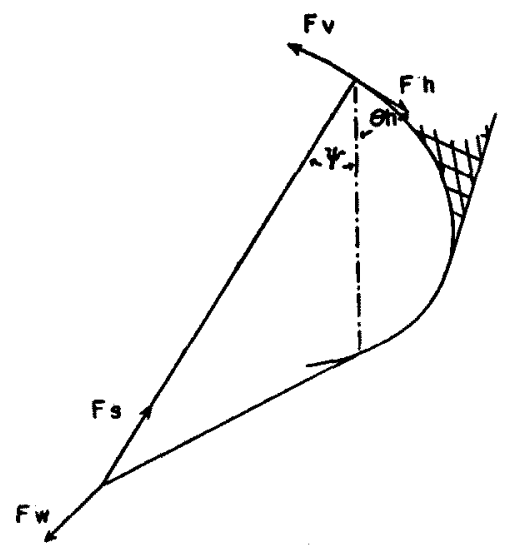

Fig. 4. Joinning part of sweep lines to the net. 


$$
F_{v}=F_{h}+F_{8}\left(\sin \theta_{h} \sin \phi \sin \phi+\cos \theta_{h} \cos \phi\right) .
$$

On the other hand, the horizontal length, $l_{y}$, from the joinning-point of upper and lower sweep lines to the central line is expressed as

$$
l_{y}=l_{s} \sin \phi \sin \phi+r_{v} \sin \theta_{v} .
$$

If we divide all the lengths by $S$, all the tensions by $T_{n}$, and all the forces by $S T_{n}$, respectively, in the preceeding expressions, we shall get 14 dimensionless relations with 15 unknown quantities. Solving these equations simultaneously, we can calculate the numerical relationships; $\left(l_{n} / S\right) \tan \theta_{n v}$ vs. $l_{y} / S$, and $\left(l_{n} / S\right) \tan \theta_{n k}$ vs. $l_{y} / S$, for different towing speeds. Thus we can predict the relation between the shape of the mouth and the towing conditions;

\section{Experimental and Results}

The model net was constructed of four pieces of tapered silk webbing. The dimensions of each part of model were as follows.

$$
\begin{array}{ll}
D=0.02 \mathrm{~cm} ., & L=0.46 \mathrm{~cm} ., \quad \varphi=30^{\circ} ., \quad S=32 \mathrm{~cm} ., \\
l_{n}=44 \mathrm{~cm} ., & l_{s}=13 \mathrm{~cm} ., \quad \text { and } \quad T_{b}=1.0 \mathrm{gr} . / 8 \mathrm{~cm} .
\end{array}
$$

The model was sustained in a current by a thin beam through the port and starboard sweep lines. A series of model experiments was carried out with different speeds of stream, varying the length of the beam, $2 l_{y}$. Then, the vertical and hori. zontal spreads of the mouth were measured, together with the resistance of the net. The comparison of the theoretical values with the experimental ones are shown in Fig. 5 .

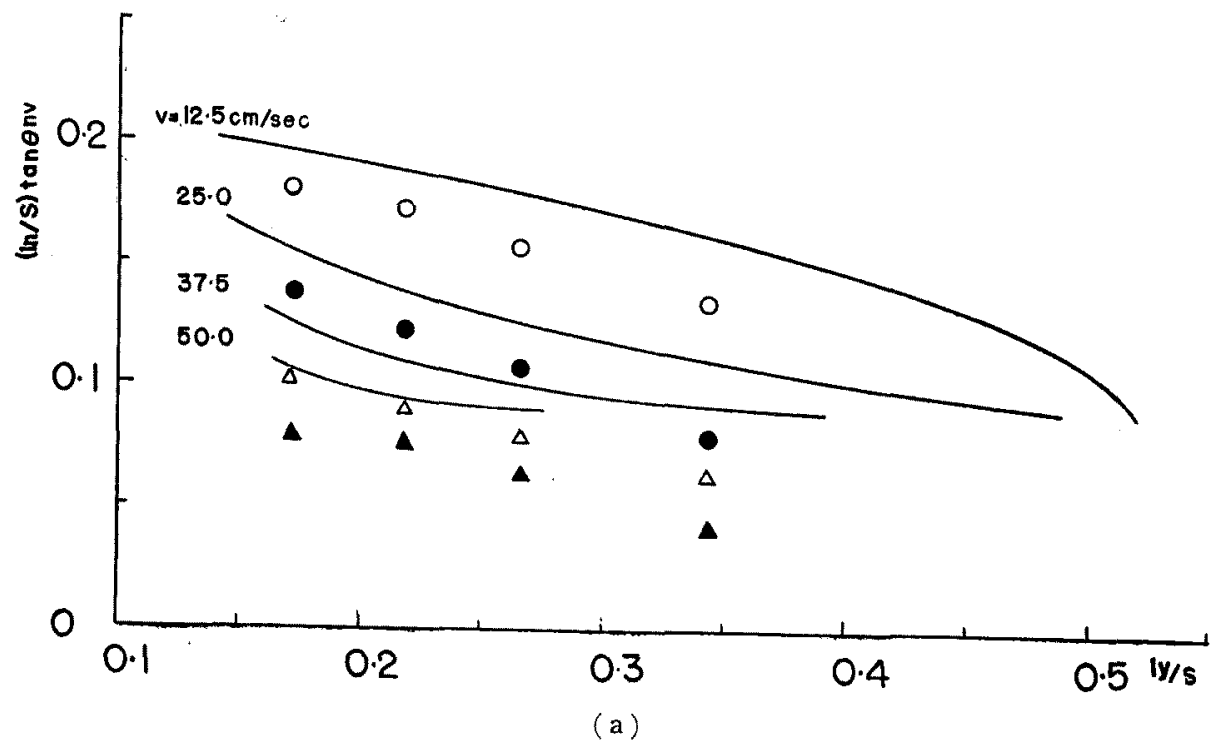




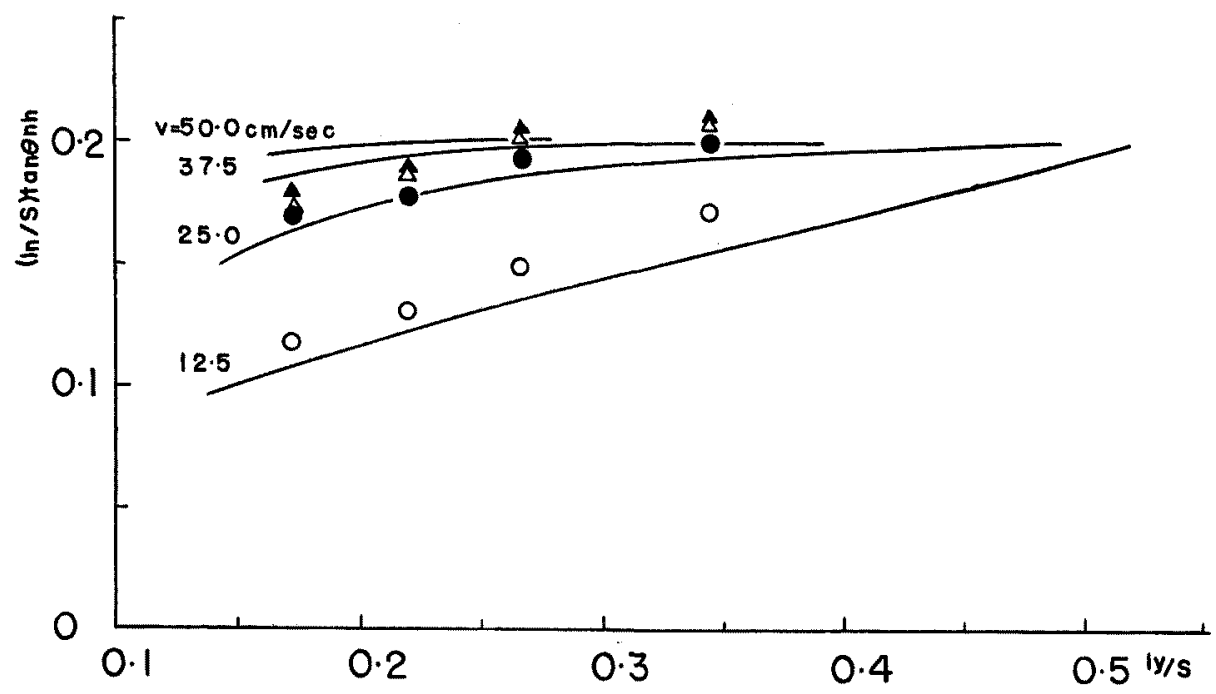

(b)

Fig. 5. Theoretical and experimental values of the relationships; $\left(l_{n} / S\right) \tan \theta_{n v}$ vs. $l_{y} / S$ and $\left(l_{n} / S\right) \tan \theta_{n h}$ vs. $l_{y} / S$. Marks, $\bigcirc, \bullet, \triangle$, and $\Delta$ denote experimental values of $V=12.5$ $\mathrm{cm} . / \mathrm{sec}$., $25.0 \mathrm{~cm} . / \mathrm{sec}$., $37.5 \mathrm{~cm} . / \mathrm{sec}$, and $50 . \mathrm{cm} . / \mathrm{sec}$., respectively.

Looking over both theoretical and experimental values, the observed values show slightly larger in horizontal spread than the theoretical. The coincidence, however, seems to be satisfactory in practical use.

The author wishes to express his deep gratitude to Prof. T. KAWAKam for his guidance and revision.

\section{References}

1) KaWAKami, T.: On the mechanical characters of the drag-net. Memoirs Coll. Agr., Kyoto Univ., No. 72, 5-15 (1955).

2) KaWAKami, T. and O. SUzuki: Studies on the kinematic behaviour of the ground rope of the trawl net-1. Bull. Jap. Soc. Sci. Fish., 25, 413-416 (1959).

3) NaKaSaI, K., O. SUzUKI and T. KaWAKami: Studies on the configuration of danish seine-1 (in Japanese). ibid, 27, 641-644 (1961).

4) TAUTI, M.: The force acting on the plane net in motion through the water. Ibid, 3, 1-4 (1934). 